\title{
River discharge simulation of a distributed hydrological model on global scale for the hazard quantification
}

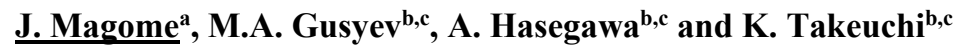 \\ ${ }^{a}$ Interdisciplinary Graduate School of Medicine and Engineering, University of Yamanashi, Japan \\ ${ }^{b}$ International Centre for Water Hazard and Risk Management (ICHARM) under the auspices of UNESCO, \\ Public Works Research Institute (PWRI), Tsukuba, Japan \\ ${ }^{c}$ National Graduate Institute for Policy Studies (GRIPS), Tokyo, Japan
}

Email: magome@yamanashi.ac.jp

\begin{abstract}
We introduce a global river discharge simulation system based on the BTOP models for individual river basins (Global BTOP) and apply Global BTOP to quantify flood and drought hazards globally. The BTOP model utilizes a modified topographic index and simulates runoff processes including snowmelt, overland flow, soil moisture in the root and unsaturated zones, sub-surface flow, and river discharge. The Global BTOP is constructed from available river network data on the 10-arcmin (about 20-km) grid in the current version. The BTOP model topographical features are obtained using river network upscaling algorithm that preserves 3-arcsec (about 90-m) HydroSHEDS and 30-arcsec (about 1-km) Hydro1K characteristics in order to reduce the impact of upscaled dataset to the discharge simulation. In the Global BTOP, the International Geosphere-Biosphere Programme (IGBP) land cover and the Food and Agriculture Organization of the United Nations (FAO) soil digital maps of the world are used for the root zone depth and soil properties, respectively. The long-term seasonal potential evapotranspiration are estimated by the Shuttleworth-Wallace model using climate forcing data CRU TS3.1 and a fortnightly Normalized Difference Vegetation Index (NDVI). The Global BTOP is run with globally available daily precipitation datasets and calibrated to the observed global and local river discharge data. From these preliminary calibration results, the Global BTOP has demonstrated good performance in selected river basins and can be utilized for many useful applications.

For the hazard quantification, we utilized simulated daily river discharges of the Global BTOP to estimate flood peak discharges of the selected return periods for the past, present and future climates. In each 10-arcmin BTOP grid, the flood peak discharges of 10-, 25-, 50-, 100- and 200-year return periods were obtained using the Gumbel distribution with L-moments. We also utilized simulated runoff of the Global BTOP to estimate standardized runoff index (SRI) for quantifying hydrologic droughts. The climate change impact assessment is conducted using GCM outputs of MRI-AGCM3.2S after applying bias correction and evaluated using flood peak discharges. For the dynamic applications of present climate, the Global BTOP is run with JAXA GSMaPNRT precipitation to simulate daily river discharges, which are utilized in a prototype near-real time discharge simulation system (GFAS-Streamflow). The GFAS-Streamflow is designed to issue flood hazard alerts globally using estimated flood peak discharges via web-interface and may also be used as a combined flood and drought hazard monitor. This may be particularly useful to close the gap between local and global scale hazard assessments under the International Flood Initiative (IFI) Flagship Project, which aims to support benchmarking flood risk reduction at global, national and local levels.
\end{abstract}

Keywords: $\quad$ River discharge, hazard assessment, flood, drought, SRI, Global BTOP 
Magome et al. River discharge simulation of a distributed hydrological model on global scale for the hazard quantification

\section{INTRODUCTION}

Many developing countries have been suffering from past flood and drought events and will likely be impacted by future hazards due to lack of preparedness and increased severity of natural hazards under climate change. The task of quantifying flood and drought hazard impacts in developing countries is challenging due to a lack of modeling technology and local hydro-meteorological data to validate these models. For the flood hazard assessment, a top-down commonly approach is adopted with river discharges simulated globally and later disaggregated to produce flood inundation maps on local and/or regional level (Gusyev et al., 2015a,b). However, there is no combined flood and drought alert system that is applied globally with local river basin details. Therefore, the quantification flood and drought risks have been attempted by the International Centre for Water Hazard and Risk Management (ICHARM) and University of Yamanashi (UY) on global and local levels with a distributed hydrological BTOP model.

In this study, we introduce a global river discharge simulation system based on the BTOP models for individual river basins (Global BTOP) and utilize simulated Global BTOP discharges to estimate flood and drought hazards globally. The simulated river discharges of the Global BTOP are combined from the simulation of 2384 BTOP models, which are developed for the individual river basins with an area of above $5,000 \mathrm{~km}^{2}$ globally. In the current Global BTOP setup, the 10-arcmin (about 20-km) grid BTOP models have been constructed with global datasets using upscaling algorithm for each individual river basin. The Global BTOP is run with regional and global meteorological forcing data such as APHRODITE V1101 (Yatagai et al., 2012) precipitation and is calibrated with available global and local river discharge data. To quantify hazards, these simulated Global BTOP discharges are utilized to obtain the flood peak discharges of 10-, 25-, 50-, 100- and 200 -year return periods using the Gumbel distribution with L-moments at each 10-arcmin BTOP grid. The hydrological droughts are quantified with standardized runoff index (SRI) using the Global BTOP simulation of runoff at each grid. In addition, the Global BTOP model is applied with GCM output data to quantify present (1979-2003) and future (2075-2099) climate change impacts as well as with near-real time satellite precipitation to issue flood hazard globally.

\section{GLOBAL BTOP SIMULATION SYSTEM}

\subsection{Block-wise TOPMODEL (BTOP)}

The core of the Global BTOP is a distributed hydrological model, Block-wise TOPMODEL (BTOP model) (Ao et al., 1999; Takeuchi et al., 1999, 2008). The BTOP model uses topographic index with blockwise concept and simulates watershed scale rainfallrunoff processes including snowmelt, overland flow, soil moisture in the root and unsaturated zones, subsurface flow, river flow routing, and dam operation (Figure 1). For the river flow routing, a modified Muskingum-Cunge (MC) routing method to conserve water at each river segment (Masutani and Magome, 2009) is also integrated. The detailed description of the BTOP model is provided by Takeuchi et al. (2008). Up to date, the BTOP model has been employed as the core hydrological module of the Yamanashi University Hydrological Model (YHyM) (Takeuchi et al., 2008) and been utilized in various hydrological applications such as large basin long-term simulations (Hapuarachchi et al., 2008), poorly or ungaugedbasins (Takeuchi et al., 1999, 2008), flood hazard assessment (Gusyev et al., 2015a,b), drought analysis with standardized indices (Gusyev et al., 2015c; Navarathinam et al., 2015), dam operation for flood and drought reduction (Navarathinam et al., 2015; Nawai et al., 2015; Odhiambo et al., 2015), nowcasting, basin-scale scenario analyses of future projection on hydro-meteorological conditions (Kiem et al., 2008) and nutrient load (Yoshimura et al., 2009).

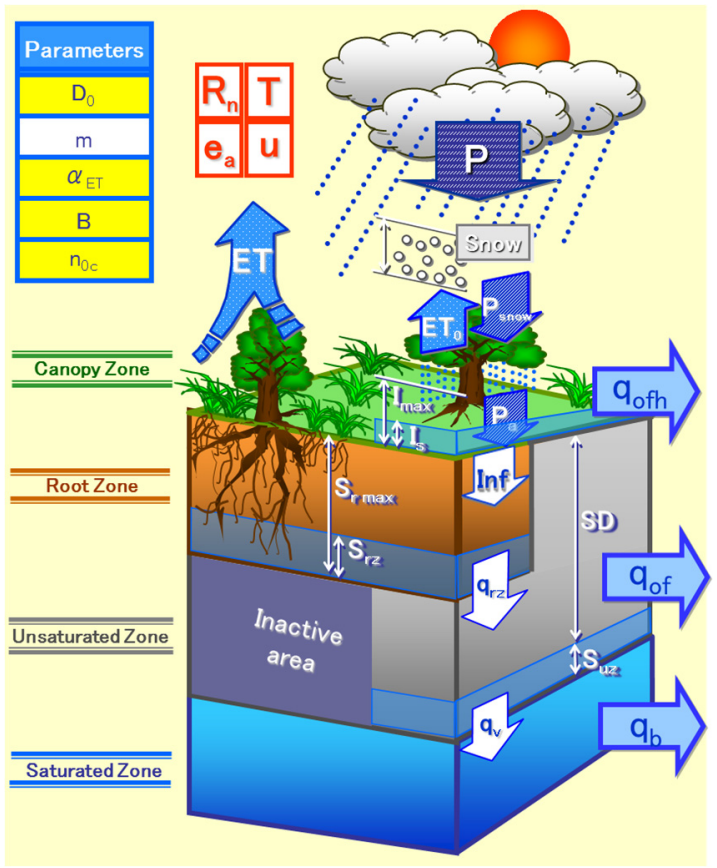

Figure 1. Schematic river basin representation in the BTOP model with BTOP grid hydrological processes. 
Magome et al. River discharge simulation of a distributed hydrological model on global scale for the hazard quantification

\subsection{Global BTOP setup}

The Global BTOP is constructed with the globally available datasets of topography, soil properties and land cover. Currently, the Global BTOP consists of 2384 river basin BTOP models developed for river basins with an area of above $5000 \mathrm{~km}^{2}$ and utilizes 10-arcmin grid resolution. The 10-arcmin river network dataset had been upscaled from 3-arcsec (about 90-m) HydroSHEDS (Lehner et al., 2008) and 30-arcsec (about 1-km) Hydro1K (USGS, 2000) datasets. To reduce the impacts of this upscaled dataset to the discharge simulation, a river network upscaling algorithm (Masutani and Magome, 2008) was applied to preserve river network features, e.g. upstream catchment area, river length and slope of original HydroSHEDS and Hydro1K data. We developed total 12 resolution levels dataset ranging from 3-arcsec to 30-arcmin grids in addition to 10-arcmin. Figure 2 shows the global 10-arcmin river network and comparison of shape of river network on different resolutions (Figure 2a-c). The International Geosphere-Biosphere Programme (IGBP) land cover of USGS GLCC V2 from LPDAAC and the Food and Agriculture Organization (FAO) digital soil map of the world (FAO, 2007) are used for the root zone depth and soil properties, respectively. For the forcing data, the longterm seasonal potential evapotranspiration (PET) is estimated by the Shuttleworth-Wallace (SW) model using climate forcing data CRU TS3.1 (CRU, 2008) and a fortnightly global Normalized Difference Vegetation Index (NDVI) dataset from Global Inventory Modeling and Mapping Studies (Tucker et al., 2004). Regional and global scale daily precipitation inputs such as APHRODITE precipitation V1101 (Yatagai et al., 2012) and CPC Unified Gauge-based Analysis of Global Daily Precipitation (Chen et al., 2008) are collected together with available local datasets and are used for calibration, validation and historical simulations of the Global BTOP.

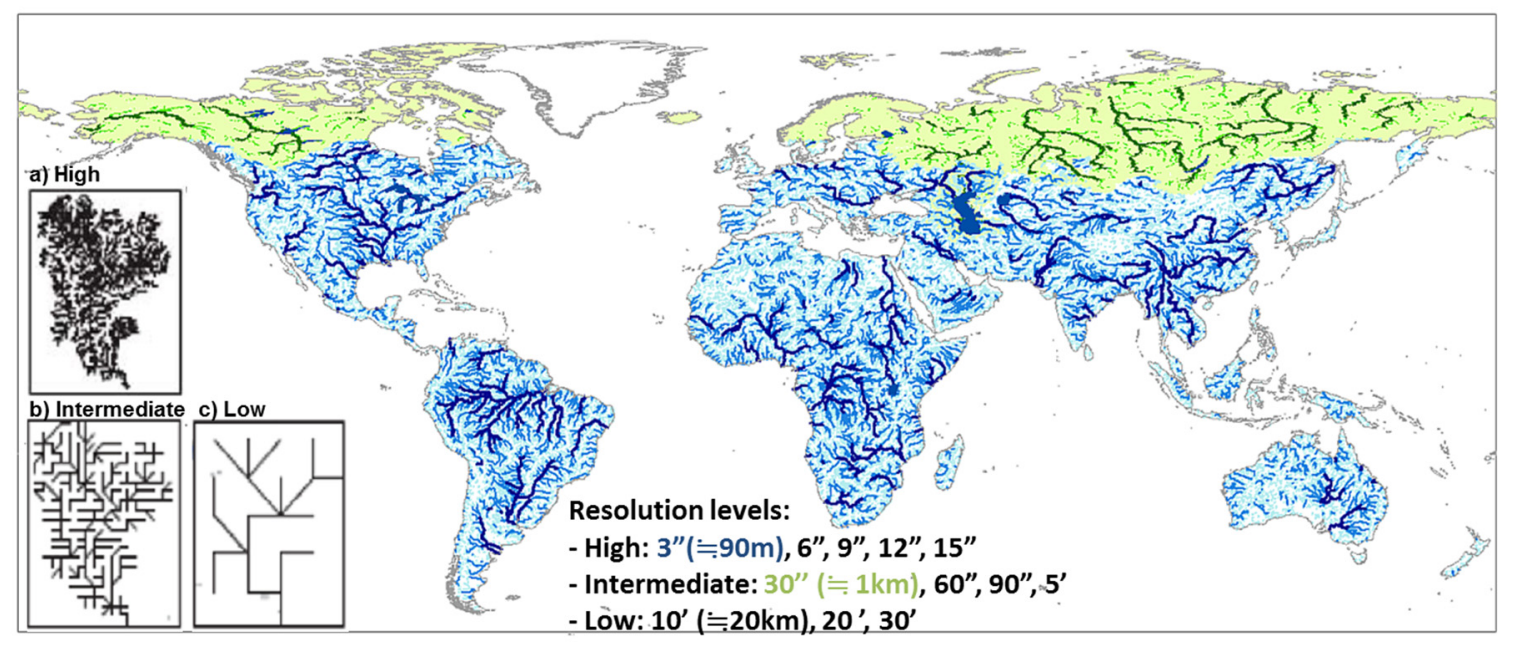

Figure 2. The Global BTOP stream networks aggregated from the original 90-m (blue) and 1-km (green) datasets. The figure insets demonstrate aggregation results for $0.5-\mathrm{km}$ (a), $5-\mathrm{km}$ (b) and $20-\mathrm{km}$ (c) levels.

\subsection{Model calibration and validation}

The Global BTOP is calibrated and validated within present period from 1979 to 2003 using time series of gauge daily discharge data obtained from Global Runoff Data Centre and local observations. For each BTOP model, the calibrated parameters are dischargeability $D$, decay factor $m$, drying function parameter $\alpha$, manning's coefficient $n_{0}$ and the groundwater parameter $b$. In this study, $b$ is kept equal to one (Takeuchi et al., 2008).

In the current global scale calibration and validation, we selected one discharge gauging station in each river basin due to limited data and poor data quality. Criterion for the selections are less number of missing value and longest record length within 1979 to 2003 in order to calibrate and validate as long as possible in present period. Figure 3 shows current target major river basins in the world and location of selected calibrated stations as well as obtained original discharge gauging station. The statistical performance of Global BTOP calibration and validation results are demonstrated in table 1 for the selected river basins.

When reasonable local discharge records are available in the basin, we conducted multiple block calibration to improve simulations, e.g. for the Mekong (Hapuarachchi et al., 2008), the Rhine (Gusyev et al., 2015a), the Ganges-Brahmaputra-Meghna (GBM) (Gusyev et al., 2015b), and the Upper Ewaso Ngiro North River (Odhiambo et al., 2015) and the Pampanga (Gusyev et al., 2015c) River Basins. We plan to follow this local calibration approach in the other river basins of the Global BTOP. 
Magome et al. River discharge simulation of a distributed hydrological model on global scale for the hazard quantification

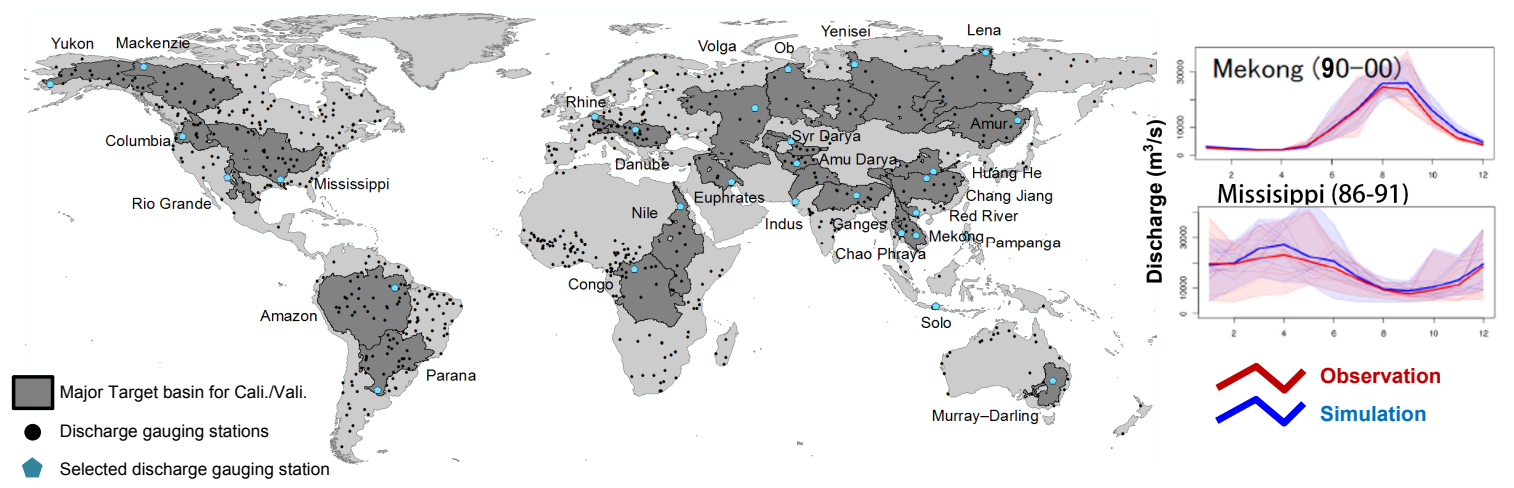

Figure 3. The Global BTOP calibration and validation at selected locations in the major river basins.

Table 1. Statistical performance of Global BTOP at selected river gauging stations

\begin{tabular}{cccccc}
\hline \multirow{2}{*}{ River basin } & \multirow{2}{*}{ Location } & \multicolumn{2}{c}{ NSE, \% } & \multicolumn{2}{c}{ Volume Error, \% } \\
\cline { 3 - 6 } & & Calibration & Validation & Calibration & Validation \\
\hline Mekong & Pakse & 86.9 & 90.3 & -4.5 & 12.6 \\
\hline Amazon & Obidos & 53.0 & 52.0 & -6.0 & 6.0 \\
\hline Mississippi & Vicksburg & 77.7 & 66.8 & 5.0 & 4.1 \\
\hline Red River & Laichau & 84.0 & 82.8 & 4.5 & -4.5 \\
\hline Ganges & Hardinge Br. & 74.0 & 67.0 & 35.0 & 19.70 \\
\hline Brahmaputra & Bahadurabad & 86.0 & 77.0 & -12.3 & -19.8 \\
\hline Meghna & Bhairab Bazar & 86.0 & 88.0 & 3.7 & -11.4 \\
\hline Rhine & Cologne & 81.0 & 85.0 & 0.6 & 2.3 \\
\hline
\end{tabular}

\section{GLOBAL BTOP APPLICATIONS}

\subsection{Flood and drought hazard assessment}

Flood hazard assessment is one of the important global applications of the Global BTOP and is conducted using flood peak discharges (Figure 4). In Figure 4, we demonstrate a global map of 50-year return period flood peak discharges from low (yellow) to high (dark blue) values. These flood peak discharges of the selected return periods such as 10-, 25-, 50-, 100- and 200-year discharge values are obtained at each 10-arcmin grid of the Global BTOP and are produced by applying the Gumbel distribution with L-moments to the Global BTOP simulated river discharges from 1979 to 2003. For the flood peak discharge comparison, those estimated flood peak discharges with 10-arcmin grid Global BTOP correlate well with the river gauging station data in the Rhine and GBM basins (Gusyev et al., 2015a,b). This comparison is currently on-going in the other river basins.

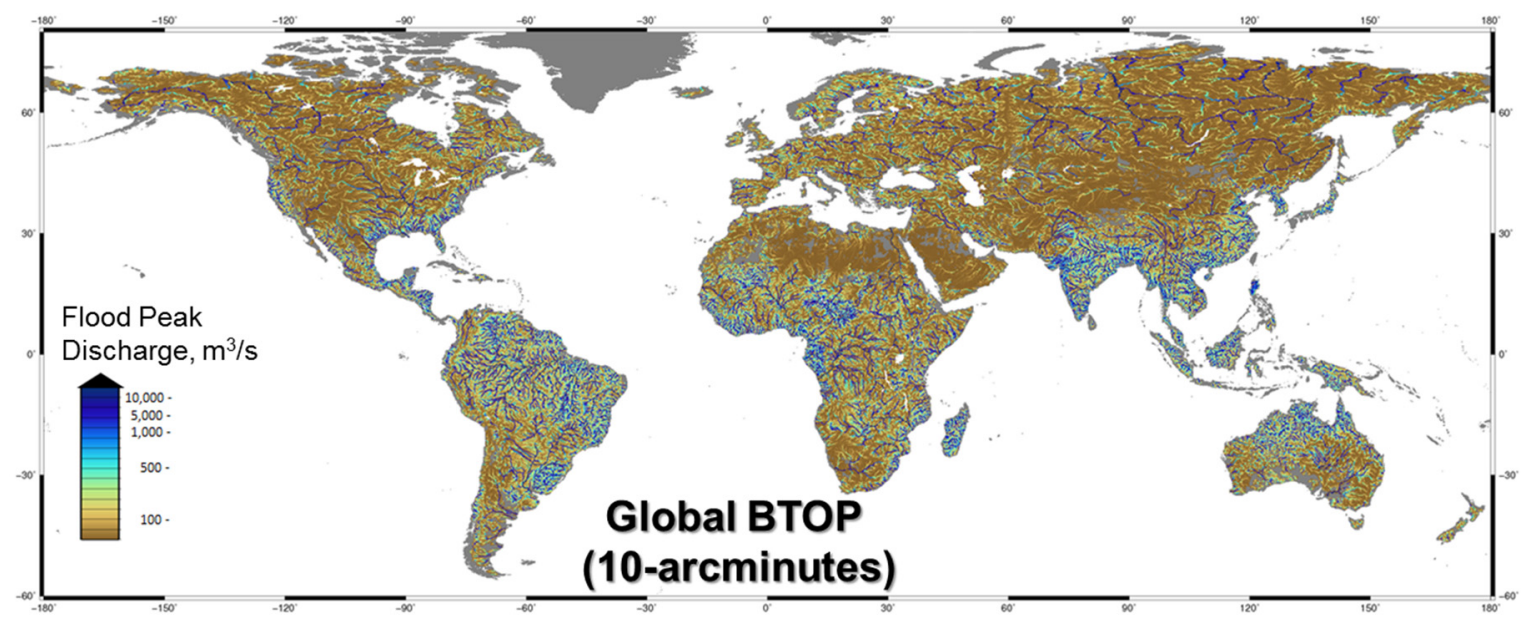

Figure 4. Estimated flood peak discharges of 50-year return period from the Global BTOP simulated river discharges at each 10-arcmin grid. 
Magome et al. River discharge simulation of a distributed hydrological model on global scale for the hazard quantification

The Global BTOP simulation results can also be utilized for the drought assessment with the standardized indices as demonstrated by Gusyev et al. (2015c). Currently, Standardized Runoff Index (SRI) (Shukla and Wood, 2008) is available from simulated runoff of the Global BTOP for the hydrological droughts. In addition, Standardized Precipitation Index (SPI) (McKee et al., 1993) is also able to calculate for evaluation of meteorological drought in the system and had been tested for historical variation on these drought indices using same data and simulated result of flood hazard assessment.

\subsection{Climate change impact assessment}

The Global BTOP is utilized for the climate change impact assessment by simulating river discharges with bias-corrected precipitation of GCM outputs. In Figure 5, the percent difference between present (1979-2003) and future (2075-2099) climates of 50-year return period flood discharge at current 10-arcmin Global BTOP grid is demonstrated using MRI-AGCM3.2S (Mizuta et al., 2012) outputs, KAKUSHIN (Mizuta et al., 2012) with SRES-A1B scenario of CMIP3 and SOSEI (Kito et al., 2015) with RCP8.5 of CMIP5. These are utilized in the Global BTOP after applying a non-parametric bias-correction method (Inomata et al., 2011; Hasegawa et al., 2015) and considered effects of different type of future scenario to simulate flood hazard in this system. From this preliminary result, the future flood peak discharges simulated with SOUSEI MRI-AGCM3.2S have larger increase over the most parts of Asia and Europe. This seems to be caused by higher extreme precipitations due to different $\mathrm{CO}_{2}$ concentration scenarios (e.g., RCP8.5 is higher than SRES-AIB).

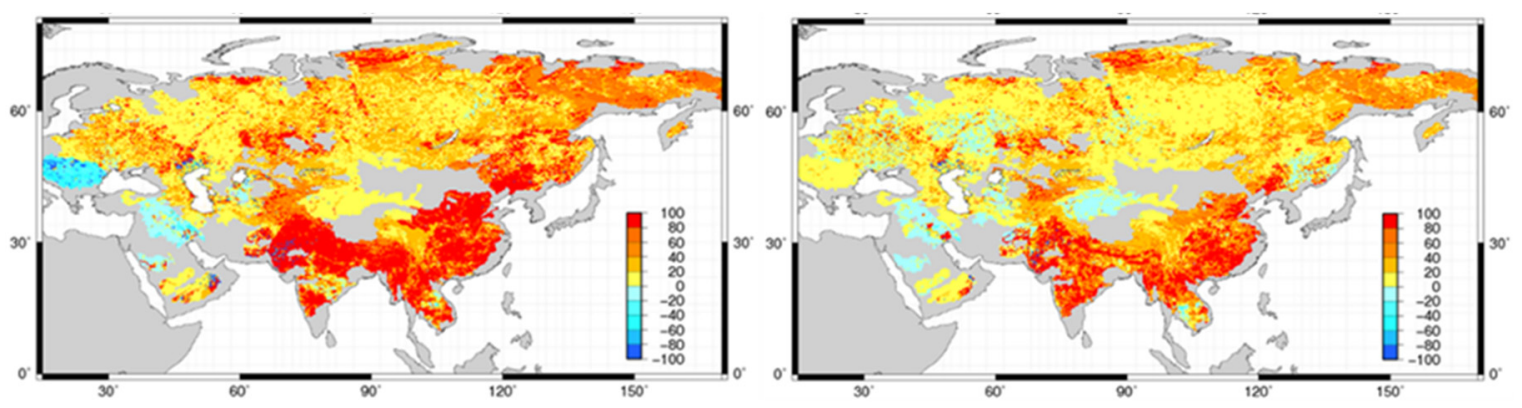

Figure 5. The percent difference of 50-year flood peak discharges between future and present climates for MRI-AGCM3.2s outputs of SOUSEI (left) and KAKUSHIN (right) projects.

\subsection{Near-Real time simulation of river discharge}

The Global BTOP has been also adopted for the daily river discharge simulation using near-real time satellite GSMaP-NRT precipitation of Japan Aerospace Exploration Agency (JAXA) GSMaP-NRT (Kachi et al., 2011) to issue flood alerts (Figure 6). The near-real time GSMaP-NRT precipitation is downloaded from JAXA (Figure 6a) and aggregated into daily values as an input for the Global BTOP. The Global BTOP uses the aggregated precipitation to simulate daily river discharges, which are compared with the flood peak discharges of 25-, 50- and 100-year return periods described in Section 3.1. From this comparison, a flood alert of high, medium and low probability floods is determined at each grid of the Global BTOP and is displayed via webinterface with blue, yellow and red colours, respectively (Figure 6b). This prototype of the flood discharge alert system is so-called Global Flood Alert System-Streamflow (GFAS-Streamflow) and utilizes a concept of the original GFAS system, which produces flood alerts based on precipitation probability estimates of the satellite precipitation data. The GFAS is operated under the framework of Infrastructure Development Institute (IDI) and International Flood Network (IFNet), Japan. Even though detailed verification process of GFASStreamflow outputs is still ongoing, a preliminary verification shows reasonable agreement of river discharge simulations with observed river discharge data and of estimated and past flood peak discharges at the study river basins. As a result, the GFAS-Streamflow may be used to issue flood alerts globally and to produce flood forecasting information and early alerts based on the simulated Global BTOP river discharge. In the future studies, the GFAS-Streamflow can be expanded to issue a combined flood and drought alerts based on the standardized indices as described in Section 3.1. 
Magome et al. River discharge simulation of a distributed hydrological model on global scale for the hazard quantification

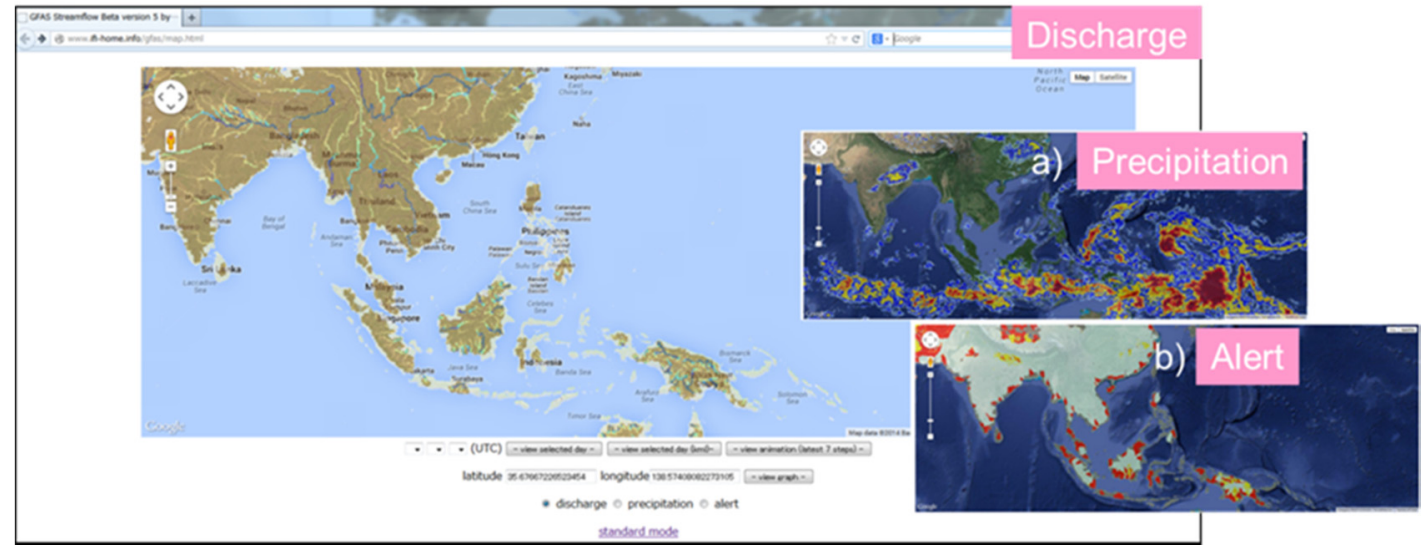

Figure 6. Global BTOP simulated river discharges from low (yellow) to high (blue) using near-real time JAXA GSMaP-NRT precipitation (a). The flood alert is shown displayed for medium and low probability flood peak discharges (b).

\section{CONCLUDING REMARKS}

This study introduced the Global BTOP river discharge simulation system and its applications for flood and drought assessment. The current version of the Global BTOP was constructed using a river network upscaling algorithm that preserves topographical features of the original 3-arcsec HydroSHEDS and 30-arcsec Hydro1K datasets to obtain current 10-arcmin grids. This technique allowed us to reduce the effect of model resolution on river discharge outputs. Even though the calibration of current Global BTOP is still on-going, the preliminary results demonstrated a good statistical performance compared to the global and local river discharge data in the various river basins around the globe. For the Global BTOP applications, simulated daily river discharges were utilized to obtain flood and drought hazards using the extreme analysis based on the Gumbel distribution with L-moments and the standardized runoff index (SRI), respectively. In addition, the climate change impact assessment was conducted using the Global BTOP with MRI-AGCM3.2S precipitation. For the dynamic applications, the Global BTOP model was utilized in a prototype near-real time discharge simulation system (GFAS-Streamflow) with JAXA GSMaP-NRT precipitation to simulate daily river discharges. As a result, the Global BTOP system demonstrated promising results with many useful applications such as issuing a combined flood and drought alerts globally.

In the future studies, we aim to reduce uncertainty of the Global BTOP results. To achieve this, a more detailed calibration and validation with block sub-division is required to improve physical representation of Global BTOP internal variables. This should result in an improvement of statistical performance compared to one block calibration procedure, especially for the large or complex river basins such as the Rhine River basin. Furthermore, a long-term simulation of Global BTOP is the key for reducing uncertainty of extreme flood peak discharges and standardized indices used for drought hazard assessment.

\section{ACKNOWLEDGEMENT}

Climate change assessment study on this work was conducted under the framework of the "Precise Impact Assessments on Climate Change" of the Program for Risk Information on Climate Change (SOUSEI Program) supported by the Ministry of Education, Culture, Sports, Science, and Technology-Japan (MEXT).

\section{REFERENCES}

Ao, T., H. Ishidaira, and K. Takeuchi (1999). Study of distributed runoff, simulation model based on block type TOPMODEL and Muskingum-Cunge method. Annual J of Hydraulic Engineering 43: 7-12 (in Japanese).

Chen, M., W. Shi, P. Xie, V. Silva, V. Kousky, R. Higgins, and J. Janowiak (2008). Assessing objective techniques for gaugebased analyses of global daily precipitation. J of Geophysical Research 113:D4

Food and Agriculture Organization (FAO) (2007). Digital Soil Map of the World version 3.6, FAO: Rome.

Gusyev, M.A., A. Gädeke, J. Magome, A. Sugiura, J. Cullmann, H. Sawano and K. Takeuchi (2015a). Connecting global and local scale flood risk assessment: A case study of the Rhine River basin flood hazard. $J$ of Flood Risk Management (resubmitted). 
Magome et al. River discharge simulation of a distributed hydrological model on global scale for the hazard quantification

Gusyev, M.A., Y. Kwak, Md. I. Khairul, Md.B. Arifuzzaman, H. Sawano, J. Magome and K. Takeuchi(2015b). Effectiveness of water infrastructure for river flood management: Part 1 - Flood Hazard Assessment using hydrological models in Bangladesh. Proc. IAHS 2015, 370, 75-81, doi:10.5194/piahs-370-75-2015

Gusyev, M.A., A. Hasegawa, J. Magome, H. Umino, and H. Sawano (2015c). Drought assessment in the Pampanga River basin, the Philippines - Part 3: Evaluating climate change impacts on dam infrastructure with standardized indices. In Proceedings of the 21st International Congress on Modelling and Simulation (MODSIM 2015), November $29^{\text {th }}$ - December $4^{\text {th }}$, Queensland, Australia.

Hapuarachchi, H.P., K. Takeuchi, M. Zhou, A.S. Kiem, M. Georgievski, J. Magome, and H. Ishidaira (2008). Investigation of the Mekong River basin hydrology for 1980-2000 using the YHyM. Hydrol Processes 22(9): 1246-1256.

Hasegawa A, Gusyev, M.A., Ushiyama T., Magome J (2015). Drought assessment in the Pampanga River basin, the Philippines Part 2: A comparative SPI approach for quantifying climate change hazards. In Proceedings of the 21st International Congress on Modelling and Simulation (MODSIM 2015), November $2^{\text {th }}$ - December $4^{\text {th }}$, Queensland, Australia.

Kachi M., T. Kubota, T. Ushio, S. Shige, S. Kida, K. Aonashi, and K. Okamoto (2011). Development and utilization of "JAXA Global Rainfall Watch" system. IEEJ Transactions on Fundamentals and Materials 131; 729-737. (In Japanese with English abstract)

Kiem, A.S., H. Ishidaira, H.P. Hapuarachchi, M. Zhou, Y. Hirabayashi, and K. Takeuchi (2008). Future hydroclimatology of the Mekong River basin simulated using the high-resolution Japan Meteorological Agency (JMA). AGCM Hydrological Processes 22(9): 1382-1394

Kitoh, A and H. Endo (2015). Changes in precipitation extremes projected by a 20-km mesh global atmospheric model, Weather and Climate Extreme (in press), doi:10.1016/j.wace.2015.09.001

Lehner, B., K. Verdin, and A. Jarvis (2008). New global hydrography derived from spaceborne elevation data. Eos, Transactions, AGU, 89(10): 93-94, http://hydrosheds.cr.usgs.gov

Masutani, K. and Magome J. (2008). Effects of the Novel Scaling Algorithm on River Networks on Discharge Simulations. Jof Japan Soc of Hydrol and Water Res 21(3): 242-247.

Masutani, K. and J. Magome (2009). An Application of Modified Muskingum-Cunge Routing Method with Water Conservation Condition to a Distributed Runoff Model, J of Japan Soc of Hydrol and Water Res 22(4): 294-300.

McKee, T.B., N.J. Doesken, and J. Kleist (1993). The relationship of drought frequency and duration to time scales. American Meteorological Society, In Proceedings of the 8th Conference on Applied Climatology 17(22): 179-183.

Ministry of Education, Culture, Sports, Science and Technology (MEXT) (2012). "Report in 2011, projection of the change in future weather extremes using super-high-resolution atmospheric models, innovative program of climate change projection for the 21st century"; Japan, March, 211 p.

Navarathinam, K., M. Gusyev, A. Hasegawa, J. Magome, and K. Takeuchi (2015). Agricultural flood and drought risk reduction by a proposed multi-purpose dam: A case study of the Malwathoya River Basin, Sri Lanka. In Proceedings of the 21st International Congress on Modelling and Simulation (MODSIM 2015), November $29^{\text {th }}$ - December $4^{\text {th }}$, Queensland, Australia.

Nawai, J., M. Gusyev, A. Hasegawa, and K. Takeuchi (2015). Flood and drought assessment with dam infrastructure: A case study of the Ba River basin, Fiji. In Proceedings of the 21st International Congress on Modelling and Simulation (MODSIM 2015), November $29^{\text {th }}-$ December $4^{\text {th }}$, Queensland, Australia.

Odhiambo, O., M. Gusyev, A. Hasegawa, J. Magome, and K. Takeuchi (2015). Flood and drought hazard reduction by proposed dams and a retarding basin: A case study of the Upper Ewaso Ngiro North River basin, Kenya. In Proceedings of the 21st International Congress on Modelling and Simulation (MODSIM 2015), November $29^{\text {th }}$ - December $4^{\text {th }}$, Queensland, Australia.

Shukla, S. and A.W. Wood (2008). Use of standardized runoff index for characterizing hydrological drought. Geophys Res Lett 35: L02405.

Takeuchi, K., T. Ao, and H. Ishidaira (1999). Introduction of block-wise use of TOPMODEL and Muskingum-Cunge method for the hydroenvironmental simulation of a large ungauged basin. Hydrol Sciences J 44(4): 633-646.

Takeuchi, K., H.A.P. Hapuarachchi, M. Zhou, H. Ishidaira and J. Magome (2008). A BTOP model to extend TOPMODEL for distributed hydrological simulation of large basins. Hydrol. Process 22: 3236-3251.

Tucker, C. J., J. E. Pinzon, and M. E. Brown (2004). Global Inventory Modeling and Mapping Studies, NA94apr15b.n11VIg, 2.0, Global Land Cover Facility, University of Maryland, College Park.

U.S. Geological Survey (USGS) (2000). HYDRO1k Elevation Derivative Database, USGS EROS Centre (available at http://eros.usgs.gov/\#/Find_Data/Products_and_Data_Available/gtopo30/hydro)

Yatagai, A., K. Kamiguchi, O. Arakawa, A. Hamada, N. Yasutomi and A. Kitoh(2012). APHRODITE: Constructing a Longterm Daily Gridded Precipitation Dataset for Asia based on a Dense Network of Rain Gauges, Bulletin of American Meteorological Society, doi:10.1175/BAMS-D-11-00122.1

Yoshimura, C., M. Zhou, A.S. Kiem, K. Fukami, H.A. Hapuarachchi, H. Ishidaira and K. Takeuchi (2009). 2020s scenario analysis of nutrient load in the Mekong River Basin using a distributed hydrological model, Science of The Total Environment 407(20): 5356-5366. 